\title{
POLE-LIKE OBJECT EXTRACTION FROM MOBILE LIDAR DATA
}

\author{
Han Zheng ${ }^{\mathrm{a}}$, Feitong Tan ${ }^{\mathrm{b}}$, Ruisheng Wang ${ }^{\mathrm{a}, *}$ \\ ${ }^{a}$ Dept. of Geomatics Engineering, University of Calgary, Calgary, Alberta, T2N 1N4, Canada - (han.zheng, ruiswang)@uclagary.ca \\ ${ }^{b}$ Yingcai Experimental School, University of Electronic Science and Technology of China, No.2006, Xiyuan Ave, West Hi-Tech, \\ Chengdu, Sichuan, P.R.China-FeitTan@outlook.com
}

\section{Commission I, ICWG I/Va}

KEY WORDS: LiDAR, Point Clouds, Local Roughness, Graph Cuts based segmentation, Pole-like object detection, DBSCAN based point clustering

\begin{abstract}
:
Object detection and recognition from LiDAR (Light Detection And Ranging) data has been a research topic in the fields of photogrammetry and computer vision. Unlike point clouds collected in well-controlled indoor environments, point clouds in urban environments are more complex due to complexity of the real world. For example, trees sometimes close to signs or buildings, which will cause occlusions in the point clouds. Current object detection or reconstruction algorithms will have problems when recognizing objects with severe occlusions caused by trees etc. In this paper, a robust vegetation removal method and a DBSCAN based pole-like object detection method are proposed. Based on observation that major difference between vegetation and other rigid objects is their penetrability with respect to LiDAR, we introduce a local roughness measure to differentiate rigid objects from non-rigid ones (vegetation in this paper). First, a local sphere with a small radius is generated for each input point. Three principal components of the local sphere are then calculated, and a plane is determined. The roughness is obtained through calculating the standard deviation of distances from all inside points to the plane by a weighted summation of the normalized distances. The further the point to the plane, the smaller the weight is. Finally, a graph cuts based method is introduced to classify the input point sets into two groups. The data term is defined by the normalized roughness of the current point, and the smoothness term is defined by the normalized distance between the point and its nearest neighbour point. In terms of pole-like object detection, first, a uniformed 2D grid is generated through projecting all the points to the XY-plane. The seed points of the pole-like objects are obtained by determining the $\mathrm{x}$ and $\mathrm{y}$ coordinates by the centres of the highest density cells of the grid and the $\mathrm{z}$ coordinate by the mean height of the point sets of each object. Finally, a DBSCAN based method is introduced to obtain the rest points of each pole-like object. Experimental results show that the proposed vegetation removal method achieves state-of-the-art results from both mobile LiDAR and airborne LiDAR data. The proposed pole-like object detection approach turns out to be very efficient.
\end{abstract}

\section{INTRODUCTION}

With the development of the laser scanning systems, and the increasing interest on three-dimensional city scene understanding and reconstruction, more and more efforts have been put on the research of object detection and recognition from LiDAR data. Existing approaches are mainly focusing on building reconstruction (Yang et al., 2013; Becker and Haala, 2007), road extraction (Zhang, 2010; Samadzadegan et al., 2009; Choi et al., 2008), pedestrian detection (Kidono et al., 2011), and road furniture detection (Guan et al., 2016). However, due to the interference of the vegetation, the results of these proposed approaches are always affected and thus in some researches, objects with interferences of vegetation are even considered as special cases (Yang et al. 2015). Existing vegetation removal methods are mainly focused on point classification through the point features such as height and local geometry information and object classification through the characteristics of points segments, such as size, shape, and orientation, by applying the heuristics or machine learning algorithms (Zhou and Neumann, 2013).

Nevertheless, as shown in Figure 1, challenges still exist when dealing with high afforestation rate area where vegetation severely interferer the object of interest (such as the street lighting pole in Figure 1). Those methods with point or object classification algorithms based on local geometry information may not work under this situation and thus result in objects undetected and misrecognition. Another challenge emerges on the object detection part. Different from the indoor scene point cloud data, mobile LiDAR data usually involves much more noises, and the point distribution are more unevenly due to the large scanning scale. Thus, those detection approaches which designed for the indoor scene can hardly be applied to the mobile LiDAR data.

In order to address the aforementioned challenges, in this paper, a robust vegetation removal method and a pole-like object detection method are introduced. It can be observed intuitively from the LiDAR data that the major difference between the vegetation and other on-ground objects (include the tree truck) is the penetrability of the object. Therefore, for a given point on an impenetrable object, its neighbour points within a sphere radius will show a regional direction consistence distribution. In contrast, the neighbour points for a given point on a penetrable object will distribute randomly inside the spherical area. Based on this idea, in this paper, a local roughness measure method is proposed to describe the points' distribution difference, thus the points are classified into two groups, then the vegetation are removed from LiDAR data. In the pole-like object detection

\footnotetext{
* Corresponding author
} 
part, the on-ground points are first detected and removed, the rest non-vegetation points are segmented into isolated point sets through the Euclidean distance clustering method. A uniformed $2 \mathrm{D}$ grid is then generated by embedding all the points into the $\mathrm{XY}$ plane. Through seeking the high density cells from the grid, the seed points of the pole-like objects are obtained. Finally, a DBSCAN based method is introduced to complement the rest points of each object.

The following part of the paper is organized as follows. Related work is reviewed in Section 2. In Section 3, details of the proposed methods are introduced. Experimental results and comparative studies are shown in Section 4. In Section 5, conclusions are drawn and the future work is discussed.

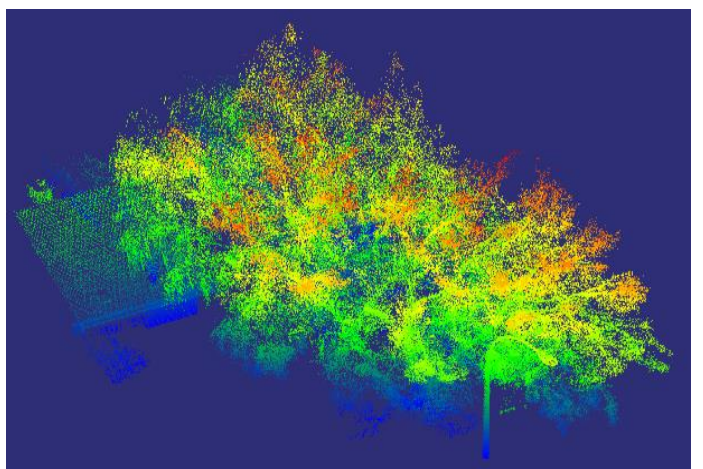

Figure 1 . Street light pole with heavy vegetation interference

\section{RELATED WORK}

We summarize the related work of vegetation removal from LiDAR data.

Vegetation removal from LiDAR data. Vegetation such as trees and bushes along with other above-ground objects such as building façade, traffic signs, and street lighting poles are the major components of a city. Most of existing researches are focusing on detecting, recognizing and modelling those manmade objects. Whereas, these methods receives acceptable results in urban area where less vegetation involves in the data. But limited results are obtained in residential area where vegetation constitutes a significant portion of the data.

In terms of vegetation detection, height(Secord and Zakhor, 2007; Yang, et al., 2015) and geometry information (Pu, et al., 2011) such as linear information are employed to segment the trees out from the LiDAR data. Instead of detecting the vegetation in a direct way, more approaches are rather introducing methods to extract objects out from the point clouds with vegetation points involved. For example the plane features employed in building façade detection.

Object detection and recognition from mobile LiDAR data. As a newly emerging technology, mobile LiDAR system has drawn much attention in the field of road inventory monitoring, 3D city model generation and so on. Recent researches (Yu et al., 2015; Huang and You, 2015) propose different methods to detect or recognize the on-ground objects with a similar pipeline which consists three key steps. 1) Segmentation. Ground points are detected and removed from the point cloud data; 2) Clustering. Based on different kinds of strategies, onground points are classified into a series of groups where each of them represent for a single object; 3) Detection and Recognition. Through applying some pre-defined features or statistical characteristics, the object-of-interest is separated from the others.

In order to recognize all the small objects in the whole city, shape features and contextual features are combined to distinguish one object from another (Golovinskiy, et al., 2009). A hierarchical detection method is introduced in (Yang, et al., 2015), the non-ground points are clustered into a series of supervoxels according to the colour and intensity information. Based on a set of predefined rules in a hierarchical order, those segments are classified into different objects. Yu et al. (2015) propose a semi-automated recognition method to extract the street light poles from the Mobile LiDAR data. The non-ground points are first segmented into small clusters through the Euclidean distance clustering method, and a normalized cut based method is employed to further segment the clusters containing more than one object. Then, the pairwise 3D shape context is generated for both the sample objects and the testing objects. At last, the street light poles are extracted through searching the matched testing objects.

Since the mobile LiDAR data mainly focusing on the street scene, objects on the ground are usually interfered by the background vegetation points (such as tree leaves), which result in the ineffectiveness of those algorithms addressing indoor scene object detection (Paul and Ramesh, 1988; Christopher and Benjamin, 2001) or outdoor objects from clean background.

\section{METHOD}

Among those on-ground objects (such as traffic sign, trees, and cars) in the point cloud which is collected from LiDAR systems, vegetation usually occupies a great portion. Furthermore, due to the characteristics of natural growth, the branches and leaves of the vegetation are growing without a regular pattern, thus result in the interference of other on-ground objects such as the situation shown in Fig. 1. As mentioned in Section 1, many algorithms are affected under this situation. In this paper, a new vegetation removal method is proposed based on the key difference between vegetation and other on-ground objects from the characteristics of regional points' distribution.
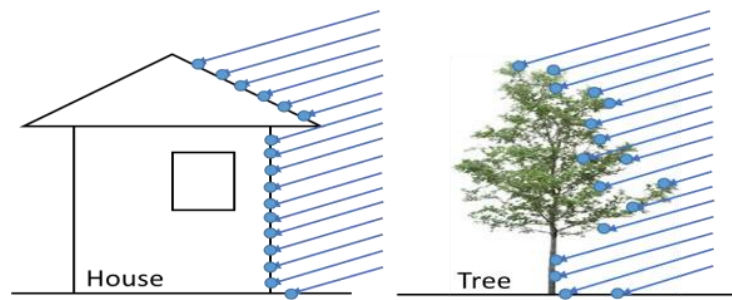

Figure 2. Schematic diagram of scanning result for impenetrable object (Left) and penetrable object (Right)

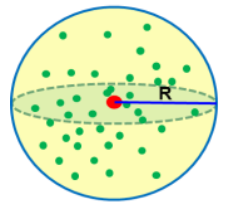

(a) Local sphere space construction

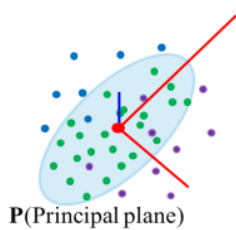

(b) Principle plane generation

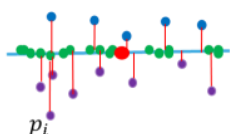

(c) Local roughness calculation
Figure 3. Schematic diagram of the construction of the local roughness 


\subsection{Local Roughness Measurement}

Based on the observation that the key difference between the vegetation and other on-ground objects (including tree trunks and branches) is the object's penetrability (as shown in Figure 2 ), we can classify the points into two different categories, the vegetation group $G(v)$ and the non-vegetation group $G(n)$, through considering the points' regional distribution with their neighbour points. As mentioned in Section 1, for those points on the impenetrable objects, their neighbour points shown a regional direction consistence distribution, while for those points on the penetrable objects, a random neighbour points' distribution will be observed.

More specifically, as shown in Figure 3, for each input point (coloured in red), a local sphere space with a small radius $\mathrm{R}$ (1.0 meter used in this paper) is assigned. Within this sphere, all the points (coloured in green) are considered to construct a covariance matrix $\mathrm{M}$ by Equation 1, and thus we can further obtain the three eigenvalues which are $\lambda_{1}, \lambda_{2}, \lambda_{3}$ by applying the principal component analysis (PCA) algorithm. After that, a principal plane $\mathrm{P}$ is obtained by the largest and the second largest principal components.

$$
M=\frac{1}{N} \sum_{i=1}^{N}\left(p_{i}-p_{C}\right) *\left(p_{i}-p_{C}\right)^{T}
$$

where $\mathrm{N}$ denotes the total point number within the sphere, $p_{i}$ denotes the $\mathrm{i}$-th $(\mathrm{i}=1,2,3, \ldots, \mathrm{N})$ point position vector $\left(x_{i}, y_{i}, z_{i}\right), p_{C}$ is the position vector of the input point.

Then distance $d_{i}$ between the point $p_{i}$ and the plane $\mathrm{P}$ is calculated. Intuitively, the more points within the sphere close to the plane $\mathrm{P}$, the more likely that the centre point (the input point) belong to the non-vegetation group. Thus we introduce the concept of 3D local space roughness of a point to describe the variation of the points within the local sphere space to the principal plane $\mathrm{P}$. The roughness of the point $\mathfrak{R}_{p_{C}}$ is calculated through Equation 2:

$$
\mathfrak{R}_{p_{C}}=\frac{1}{N} \sum_{i=1}^{N}\left(1-e^{-\alpha \frac{d_{i}}{R}}\right)
$$

where $\alpha$ is a constant which used to increase the range of $d_{i} / R$ from $[0,1]$ to $[0, \alpha]$. Thus the roughness of the point is normalized.

\subsection{Graph cuts based points classification}

Based on the roughness obtained through the method introduced in section 3.1, the points' labels can be assigned with a data energy term $D_{p}\left(f_{p}\right)$ from the energy minimization perspective as:

$$
D_{p}\left(f_{p}\right)=\frac{2}{\pi} \arctan \left(-\log _{2} \Re_{p_{\mathrm{C}}}\right)
$$

where $f_{p}$ denotes the label of the point $\mathrm{p}$ is assigned as foreground non-vegetation cluster.
While in order to construct the smoothness term $V_{p, q}\left(f_{p}, f_{q}\right)$ (as shown in Equation 4), a kd-tree is first generated and the neighbouring point pairs is defined based on the nearest points' distance, thus a nearest distance based graph is generated.

$$
V_{p, q}\left(f_{p}, f_{q}\right)=e^{-\left(\frac{\left|p_{i}-p_{j}\right|}{\sigma}\right)^{2}}
$$

where $p_{i}$ and $p_{j}$ are the two points in the point pair, $\sigma$ denotes the variance of the point pair's distance.

Through combining the two energy terms shown in Equation 3 and Equation 4, we build the energy function as:

$$
E(f)=\sum_{p \in G} D_{p}\left(f_{p}\right)+\sum_{\{p, q\} \in G} V_{p, q}\left(f_{p}, f_{q}\right)
$$

where $\mathrm{G}$ denotes the point group of a cluster.

By applying the well-known graph-cut method to minimize the energy function, the points are classified into two categories, the foreground non-vegetation point set, and the background vegetation point set.

Figure 4 shows the result of the introduced vegetation removal method. As seen from Figure 4, points belong to the tree leaves are removed, while the street light pole, tree trunks and the façade of the house are left. After removed the tree leaves, the street lighting pole is isolated from other objects, it will contributes to better object detection and recognition results.
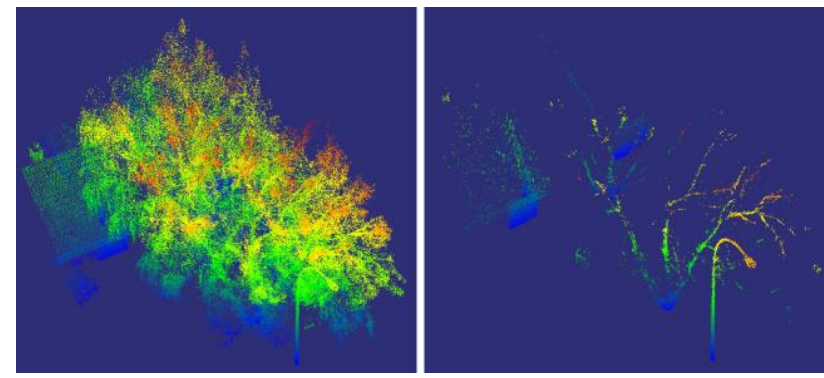

Figure 4. Before (Left) and after (Right) the introduced vegetation removal method apply to the point clouds.

\subsection{DBSCAN based pole-like objects extraction}

Intuitively, pole-like objects share a vertical upward characteristic. Thus, it is easy to locate the position of the polelike objects through exploring the cells with high densities after embedding all the points into the 2D uniformed grid. Based on this idea, for the non-vegetation point cloud, a 2D image where each cell represents one pixel, and the intensity value of each pixel is obtained through counting the number of the points inside the cell. By applying a predefined intensity threshold to the image, the positions of the pole-like objects on XY-plane are obtained.

To extract the pole-like object from the non-vegetation point cloud, in this paper, a DBSCAN (Ester, et al., 1996) based method is introduced. 
The seed points are chose for each pole-like object based on the obtained $2 \mathrm{D}$ positions. In order to avoid the influence from the remained on-ground points, the $\mathrm{Z}$ coordinate is obtained by calculating the mean $\mathrm{Z}$ coordinate value of all the points inside the cell. Once the seed point is obtained, the DBSCAN based method is applied to find the rest points belongs to the object.

Again, a small radius $r$ is set for the local sphere space construction. Start from the seed point, we generate a local sphere space centred at the point. Within the sphere, if the point number is larger than a predefined threshold, all the points inside of the sphere are added to the object points queue Q. Then for every unsearched point in Q, apply the same operation as the seed point, until all points in $\mathrm{Q}$ are searched. The polelike object clusters are obtained when the search finished.

\section{EXPERIMENTAL RESULTS}

\subsection{Vegetation Removal}

The introduced vegetation removal method is tested on 2 datasets which involves both airborne LiDAR data and mobile LiDAR data. The airborne LiDAR dataset which covers an area in City of Vaihingen, Germany is sourced from ISPRS Test Project. With different kinds of area (river, farmland, residential area, playground and grassland) included, the dataset contains some 3.8 million points. While, the mobile LiDAR dataset which contains over 20 million points is provided by the Teledyne Optech Corporation. The point cloud is collected in a residential area along a street, the point density along the road is about 1019.2 points $/ \mathrm{m}^{2}$. Some of the experimental results are shown in Figure 5 and Figure 6. The overall results of the two datasets are shown in Figure 9 and Figure 10, respectively.

In Figure 5, the vegetation removal results with four typical scenes involves vegetation are demonstrated. The source point clouds are shown in the left column in Figure 5, while the corresponding vegetation removal results are shown in the left column in Figure 5 with the extracted vegetation points coloured in green and the remaining points (represent for the flat area) coloured in blue. As seen from the vegetation removal results in Figure 5(b), 5(d), 5(f) and 5(h), the tree leaves, grass, bushes and even the standing grain are detected and segmented out with the proposed vegetation removal method applied. However, the roofs, road and the ground after harvest are well preserved. Although a few non-vegetation points may be detected as the vegetation points (such as the green points on the farmhouse's roof at the bottom of Figure 5(h)) in the flatness area, the influence for the overall result is extremely limited.

Two typical scenes with vegetation involves in mobile LiDAR data in residential area are shown in Figure 6. Also, the left column and the right column are the source point clouds and the vegetation removal results, respectively. In the vegetation removal results, the segmented out vegetation points are coloured in green, and the remaining points are coloured in orchid. As seen from Figure 6(b) and 6(d), vegetation points are extracted, the ground, building façade, and even the pole-like objects are well preserved. Also, false positive points may still be observed in some area (such as the green points on the building façade on both sides of the road in Figure 6(b)). The consequence is caused by the uneven surface of the house façade. As seen from Figure 7, the corner area of the wall and the area of window edges are the main areas where the false positive points be spotted. Better results may obtain through decreasing the radius of the local sphere space.

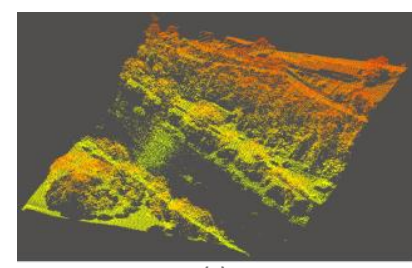

(a)
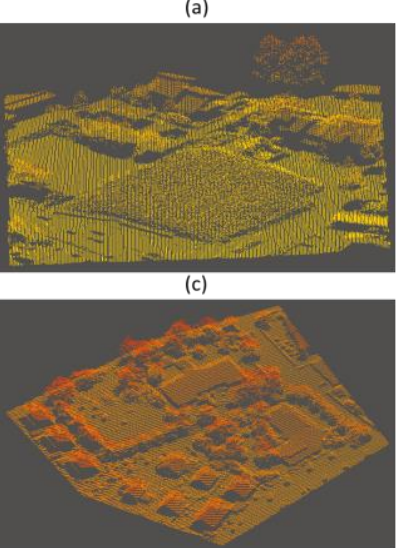

(e)

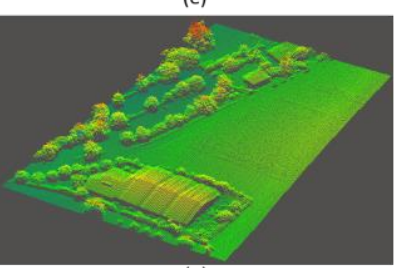

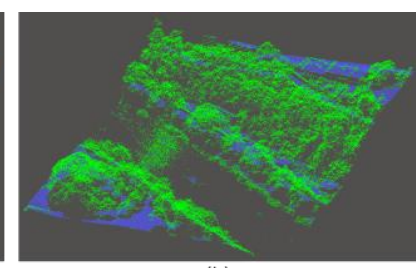

(b)

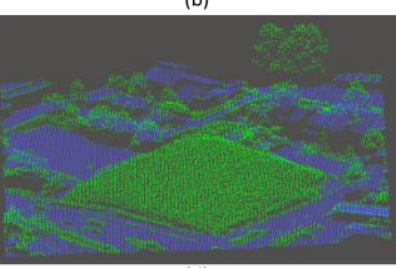

(d)
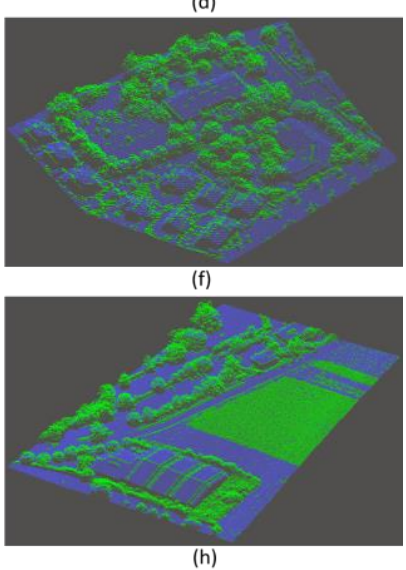

Figure 5. Experimental results of the airborne LiDAR dataset. (a) The source point cloud, and (b) the vegetation removal result of the banks along the river. (c) The source point cloud, and (d)

the vegetation removal result of a grass land area. (e) The source point cloud and (f) the vegetation removal result of a residential area. $(\mathrm{g})$ The source point cloud and $(\mathrm{h})$ the vegetation removal result of a farmland area.

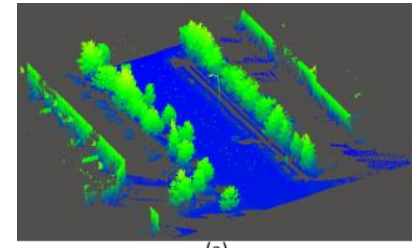

(a)

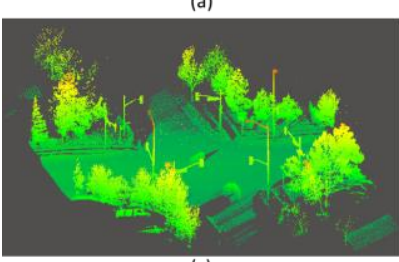

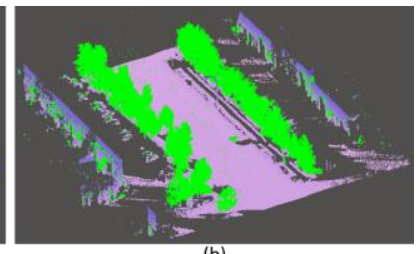

(b)

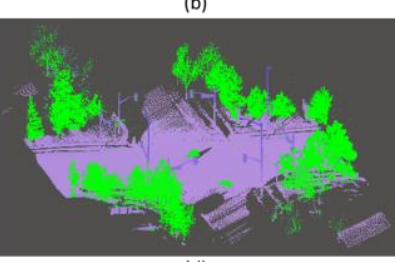

(d)
Figure 6. Experimental results of the mobile LiDAR dataset. (a) The source point cloud, and (b) the vegetation removal result of an area along the road. (c) The source point cloud, and (d) the vegetation removal result of a crossroad.

\subsection{Pole-like Object Extraction}

As mentioned in section 2, the above ground objects are always interfered by the vegetation. After removed the vegetation points, the surroundings of the above ground objects are cleaner. 
Figure 8 shows a step by step pole-like objects extraction progress from the mobile LiDAR dataset. As seen from Figure 8, after removed the vegetation points, above ground objects are isolated with each other, with the DBSCAN based extraction method, pole-like objects are extracted completely and accurately.
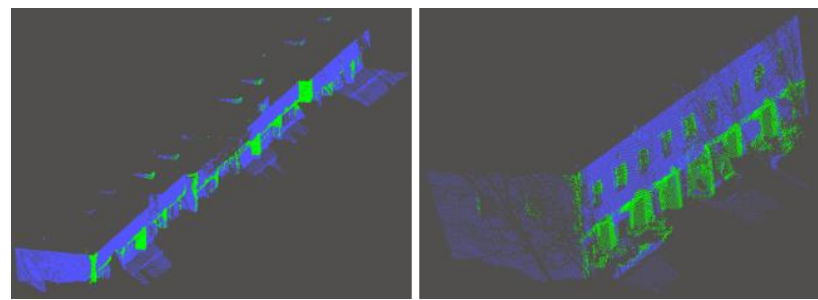

Figure 7. Close view of the false positive points (coloured in green) area on building facade.
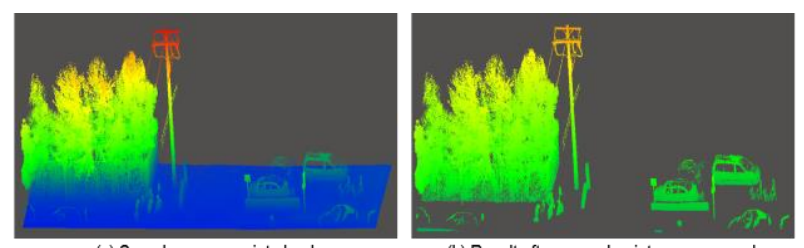

(a) Sample source point cloud
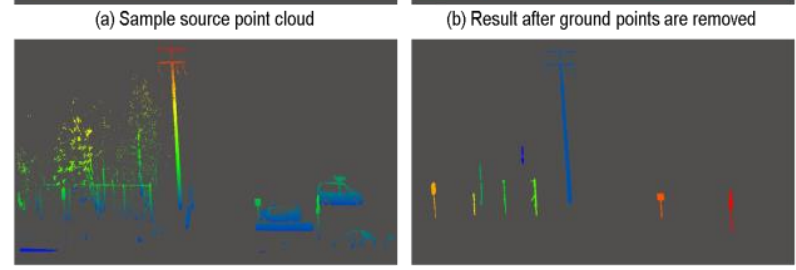

(c) Result after the vegetation removal method applied (d) Result of the Pole-like objects extraction method

Figure 8. A step by step result of a sample point cloud with the proposed methods applied.

\section{CONCLUSION}

In this paper, a novel vegetation removal method and a DBSCAN based pole-like object extraction method are introduced. Based on the observation that the key difference between the vegetation and other rigid objects in point cloud data is their penetrability, a local roughness measure is defined and applied to describe the difference of points' distribution in the local space between the two object categories. Based on the roughness measure, an energy function is built afterwards. Through minimizing the energy function by the Graph-cuts based method; the input point cloud is segmented into two groups, the vegetation point set and the non-vegetation point set Experimental results show that the proposed vegetation removal method receives significant results in both mobile LiDAR data and airborne LiDAR data. In terms of pole-like object extraction, a DBSCAN based method is introduced. Experimental results show that the pole-like objects are extracted completely and accurately. Since the pole-like objects are extracted from the point cloud, further researches will focus on pole-like object recognition and modelling.

\section{REFERENCES}

Bisheng Y., Zheng Wei, Qingquan Li, and Jonathan Li, 2013. Semiautomated building façade footprint extraction from mobile Lidar point clouds, IEEE Geoscience and Remote Sensing Letters, 10(4), pp. 766-770.

Christopher M. C. and Benjamin B. Kimia, 2001. 3D object recognition using shape similiarity-based aspect graph, Eighth
IEEE International Conference on Computer Vision, 1, pp. 254-261.

Ester, M., Hans-Peter Kriegel, Jörg Sander, and Xiaowei Xu, 1996. A density-based algorithm for discovering clusters in large spatial databases with noise. Proceedings of $2^{\text {nd }}$ International Conference on Knowledge Discovery and Data Mining. 96(34), pp. 226-231.

Farhad S., Michael Hahn, and Behnaz Bigdeli, 2009. Automatic road extraction from LiDAR data based on classifier fusion, IEEE in Urban Remote Sensing Event, pp. 1-6.

Haiyan G., Yongtao Yu, Jonathan Li, and Pengfei Liu, 2016. Pole-like object detection in mobile LiDAR data via supervoxel and bag-of-contextual-visual-words representation, IEEE Geoscience and Remote Sensing Letters, 99, pp. 1-5.

Jing H., and Suya You, 2015. Pole-like object detection and classification from urban point clouds, IEEE International Conference on Robotics and Automation, pp. 3032-3038.

Kiyosumi K., Takeo Miyasaka, Akihiro Watanabe, Takashi Naito, and Jun Miura, 2011. Pedestrian recognition using highdefinition LiDAR, IEEE Intelligent Vehicles Symposium (IV), Baden, Germany, pp. 5-9.

Paul J. B. and Ramesh C. Jain, 1988. Segmentation through variable-order surface fitting, Pattern Analysis and Machine Intelligence, IEEE Transactions on, 10(2), pp. 167-192.

Qianyi Z. and U. Neumann, 2013. Complete residential urban area reconstruction from dense aerial LiDAR point clouds, Graph. Models, 75(3), pp. 118-125.

Secord J., and Avideh Zakhor, 2007. Tree detection in urban regions using aerial lidar and image data. Geoscience and Remote Sensing Letters, IEEE, 4(2), pp. 196-200.

Shi P., M.R., George Vosselman, Sander Oude Elberink, 2011. Recognizing basic structures from mobile laser scanning data for road inventory studies, ISPRS Journal of Photogrammetry and Remote Sensing, 66(6), pp. S28-S39.

Susanne B., Norbert Haala, 2007. Refinement of building fassades by integrated processing of Lidar and image data, International archives of photogrammetry, remote sensing and spatial information sciences, 36, pp. 7-12.

Wende Z., 2010. LiDAR-based road and road-edge detection, IEEE Intelligent Vehicles Symposium, pp. 845-848.

Yun-Woong C., Young-Woon Jang, Hyo-Jong Lee, and Gisung Cho, 2008. Three-dimensional LiDAR data classifying to extract road point in urban area, IEEE Geoscience and Remote Sensing Letters, 5(4), pp. 725-729.

Bisheng Y., Zhen Dong, Gang Zhao, and Wenxia Dai. 2015. Hierarchical Extraction of Urban Objects from Mobile Laser Scanning Data. ISPRS Journal of Photogrammetry and Remote Sensing 99(1), pp. 45-57.

Yongtao Y., Jonathan Li, Haiyan Guan, Cheng Wang and Jun Yu, 2015. Semiautomated Extraction of Street Light Poles From Mobile LiDAR Point-Clouds, IEEE Trans. Geosci. Remote Sens., 53(3), pp. 1374-1386. 


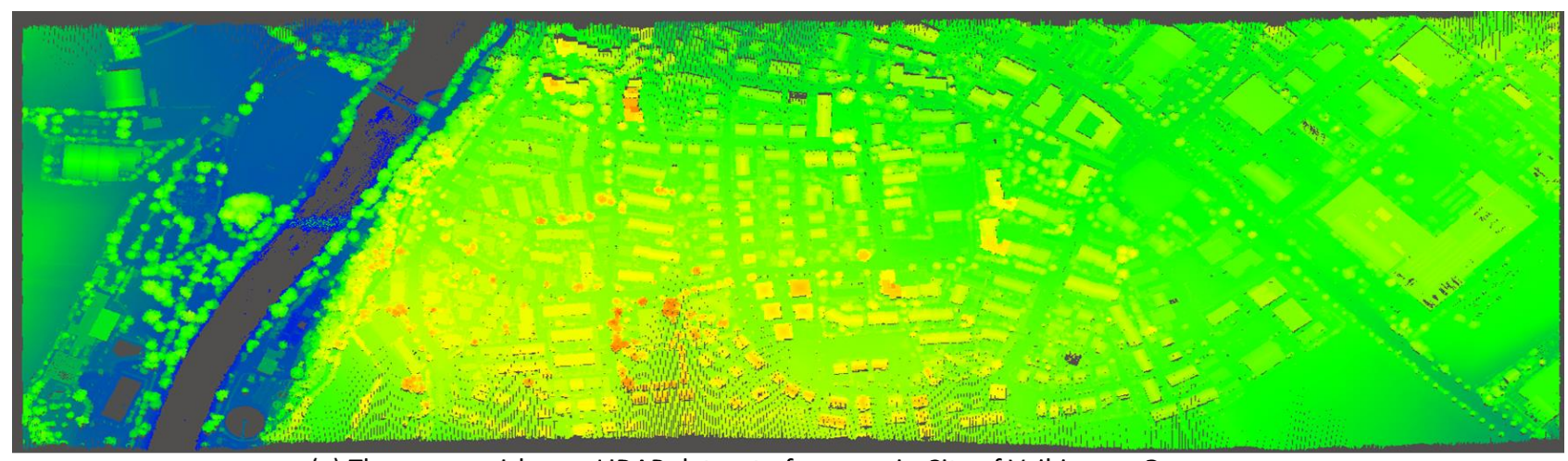

(a) The source airborne LiDAR dataset of an area in City of Vaihingen, Germany

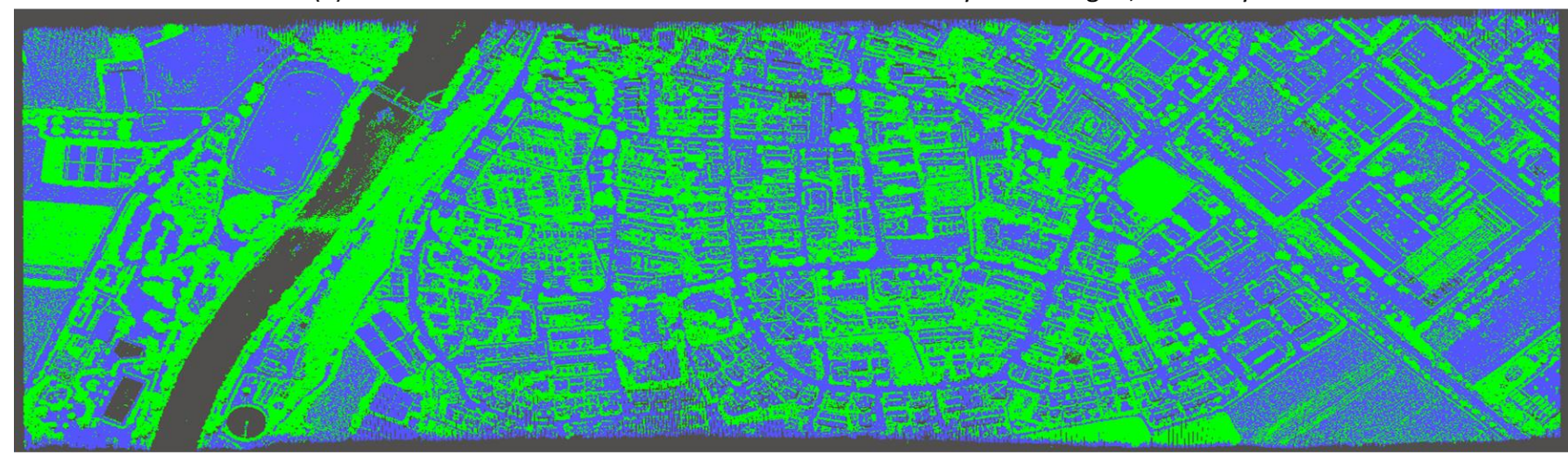

(b) The vegetation removal results of the airborne LiDAR dataset

Figure 9. The overall vegetation removal results of the airborne LiDAR dataset.

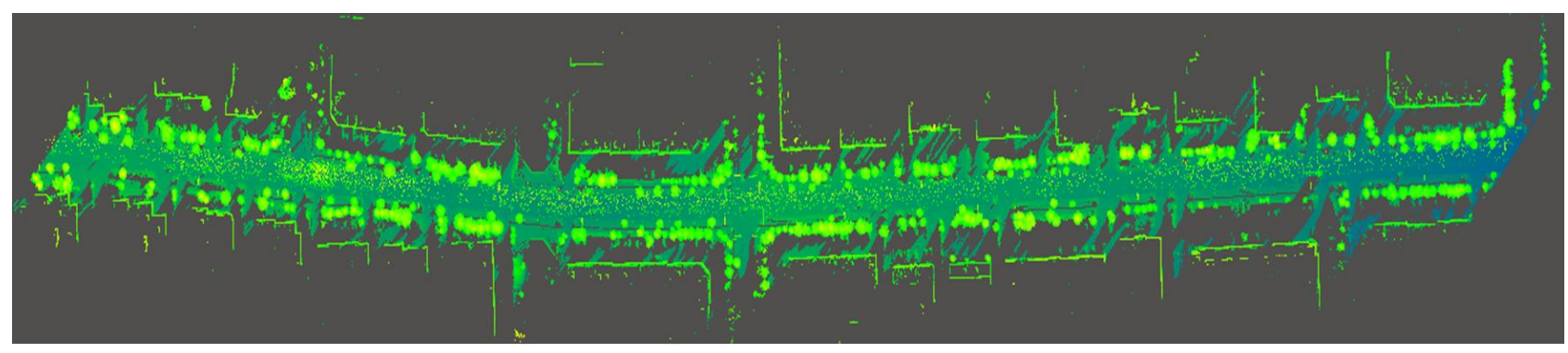

(a) The source mobile LiDAR dataset of an street in the residential area

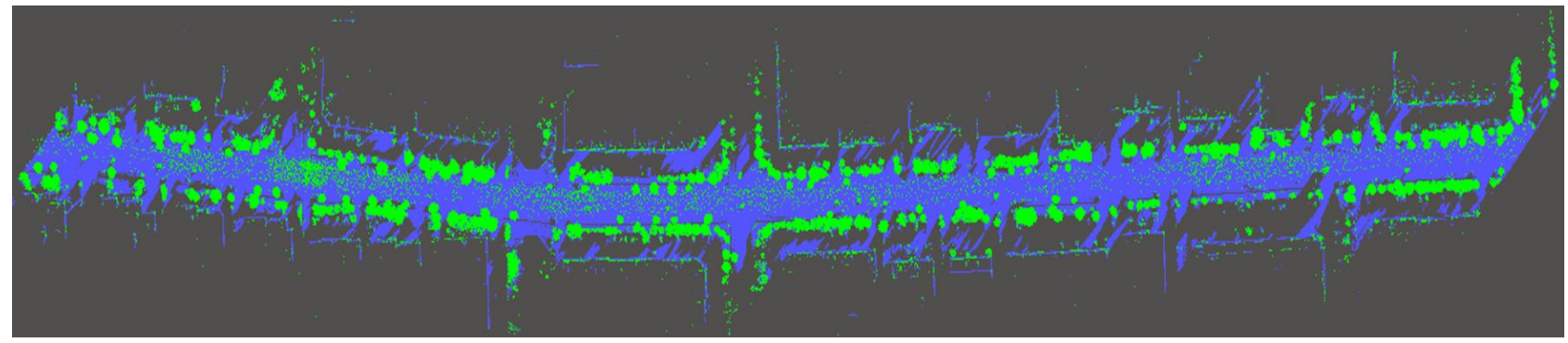

(b) The vegetation removal results of the mobile LiDAR dataset

Figure 10. The overall vegetation removal results of the mobile LiDAR dataset. 